Daisuke Miyazaki, Masataka Kagesawa, Katsushi Ikeuchi, "Transparent Surface Modeling from a Pair of Polarization Images," IEEE Transactions on Pattern Analysis and Machine Intelligence, Vol.26, No.1, pp.73-82, 2004.01 



\title{
Transparent Surface Modeling from a Pair of Polarization Images
}

\author{
Daisuke Miyazaki Masataka Kagesawa \\ Katsushi Ikecuhi, Fellow, IEEE \\ Institute of Industrial Science, The University of Tokyo, \\ 4-6-1 Komaba, Meguro-ku, Tokyo, Japan 153-8505, \\ E-mail: \{miyazaki, kagesawa, ki\}@cvl.iis.u-tokyo.ac.jp, \\ IEEE Transactions on Pattern Analysis and Machine Intelligence, \\ Vol.26, No.1, January 2004, pp.73-82
}

\begin{abstract}
We propose a method for measuring surface shapes of transparent objects by using a polarizing filter. Generally, the light reflected from an object is partially polarized. The degree of polarization depends upon the incident angle which, in turn, depends upon the surface normal. Therefore, we can obtain surface normals of objects by observing the degree of polarization at each surface point. Unfortunately, the correspondence between the degree of polarization and the surface normal is not one to one. Hence, to obtain the correct surface normal, we have to solve the ambiguity problem. In this paper, we introduce a method to solve the ambiguity by comparing the polarization data in two objects, i.e., normal position and tilted with small angle position. We also discuss the geometrical features of the object surface and propose a method for matching two sets of polarization data at identical points on the object surface.
\end{abstract}

Keywords: I.4.8.j Shape, I.5.4.b Computer vision.

\section{Introduction}

Recently, techniques for 3D modeling of objects through observation have been extensively investigated. Such 3D modeling has a wide range of applications, including virtual reality and object recognition. Geometry is one 


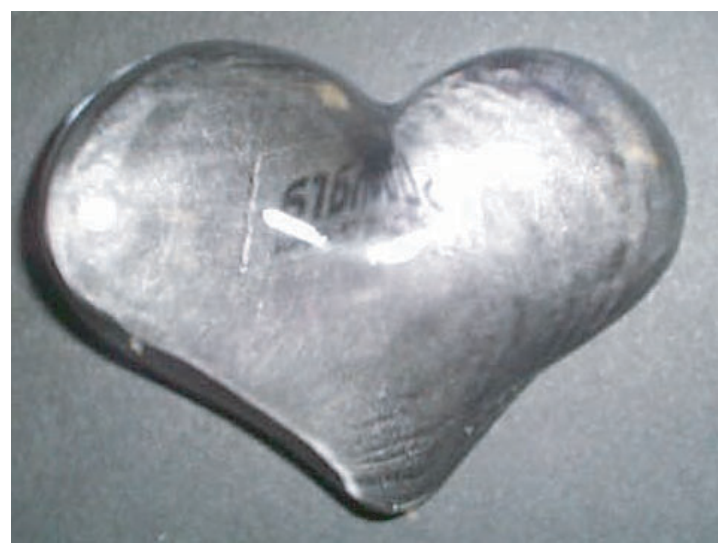

(a)

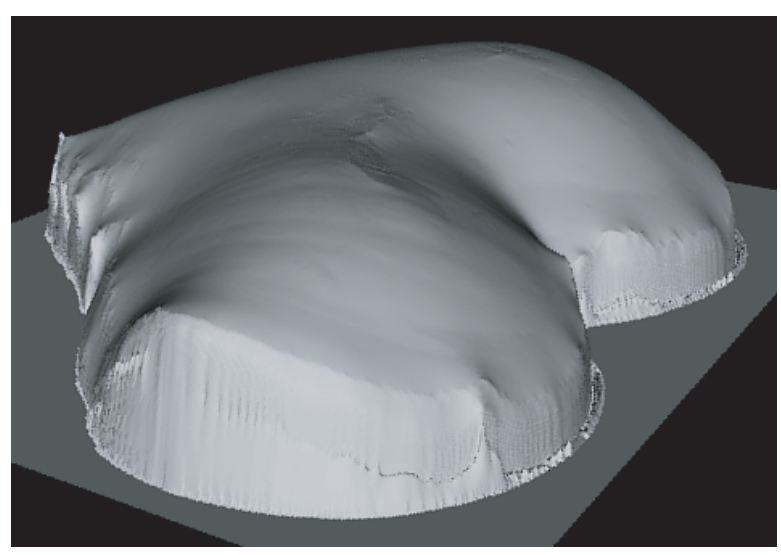

(b)

Figure 1: (a) Photograph of transparent heart-shaped glass and (b) the obtained shape of this object.

of the most important aspects of 3D modeling since we can generate realistic images from geometrical models obtained by measuring the shape of an object.

The computer vision community has extensively developed techniques to determine the shape of objects[1,2]. Most of these methods are, however, designed to obtain shapes of opaque unhomogeneous surfaces, namely, the analysis of these methods is based on the body reflection component of object surface reflections. Models of transparent objects as well as those of black objects and metals cannot be created using those techniques since they have surface only reflection. In this paper, we propose a method for obtaining the surface shape of transparent objects. Fig. 1a is a picture of a heart-shaped piece of glass and Fig. $1 \mathrm{~b}$ is the shape of the object estimated by our method.

\section{$1.1 \quad$ Related Work}

Many methods have been developed to deal with transparent objects. Szeliski et al.[12] and Schechner et al.[13] separated surface reflection of a transparent planar object from background images. Zongker et al.[14], Chuang et al.[15], Wexler et al.[16], and Matusik et al.[17] developed a method to generate the appearance of a transparent object from a series of images taken under different conditions. These methods, however, do not totally provide the shape information of the transparent object.

Few existing methods attempt to determine object shape through surface 
reflection. Ikeuchi[3] proposed a method to determine the shape of a metal surface by using photometric stereo. Nayar et al.[4] extended the method by using continuous illumination distribution, referred to as a photometric sampler. Sato and Ikeuchi[5] analyzed color images in a similar setting and determined the shape and reflectance of shiny objects. Oren and Nayar[6] proposed a method using surface reflections and motion to determine surface shape.

Surface reflection can also be analyzed through the degree of polarization, as demonstrated by Koshikawa and Shirai[7, 8], who employed polarized light sources to determine the shape of planar metal surfaces. Wolff and Boult $[9,10]$ proposed a method to obtain the surface normal of planar metals and glasses by analyzing the polarization of the object. Rahmann and Canterakis[11] proposed an optimization framework for shape recovery and recovered the spherical shape of an opaque specular object by using five polarization images. Saito et al.[18] employed the analysis of the degree of polarization and developed a method with which the surface of a transparent object could be determined; however, the degree of polarization provided two candidates of surface normal, and they did not solve this ambiguity. Miyazaki et al.[19] solved this ambiguity problem by introducing the degree of polarization of thermal radiation in the infrared wavelength and uniquely determined the surface orientation.

In this paper, to resolve the ambiguity problem, we introduce polarization analysis from two views. This method obtains two sets of data of polarization from two different views. We observed the object twice from the same camera by tilting the object at a small angle. This method first obtains the degree of polarization, as in the method proposed by Saito et al.[18]. One measurement of the degree of polarization corresponds to two surface orientations. The degree of polarization of a novel view disambiguates this problem. By comparing the degree of polarization at the same surface point (= corresponding point) of each piece of polarization data, we can determine the unique surface orientation.

\subsection{Assumptions}

There are several assumptions we use to successfully apply our method:

1 The object is observed as an orthographic projection to the image plane of the camera.

2 The object is transparent and solid.

3 The refractive index is known and constant for any part of the object. 
4 The object surface is optically smooth (not microscopically rough).

5 The object surface is geometrically smooth and closed ( $C^{\infty}$ surface).

6 No self-occlusion exists. There is no "jump" in the height of the object surface.

7 The entire frontal surface is included in the camera field of view.

8 The light that is directly reflected from the object surface should be observed. The light that is reflected two or more times or is transmitted should not be observed.

9 The disambiguation method of the azimuth angle $\phi$ shown in Section 2.1 can be applied.

10 The light caused by interreflection is unpolarized and uniform for all the points on the object surface.

11 Hints for region segmentation are given by the human operator.

12 The class of regions does not change even if we rotate the object at a small angle.

13 Proper rotation direction is given by the human operator.

14 The object still obeys all these assumptions even if we rotate the object at a small angle.

In fact, assumption 2 is not mandatory. Our method is also effective for opaque objects.

Several kinds of concave objects violate assumption 8 and produce a less precise shape of the object. From assumption 9, objects which have complete concave parts - a surface which is always concave for any direction, e.g., dimples - cannot be modeled automatically by our method.

We rotate the object to obtain the polarization data observed from two different views. Our method assumes that there are no interreflections and that the rotation angle of the object is infinitesimal. However, under certain conditions, interreflections cannot be avoided. From assumption 10, we subtract the intensity caused by interreflection from the input data to reduce the interference of interreflection. We consider that such modification empirically produces a more satisfactory data than does input raw data; however, such assumptions are theoretically not always true.

Our method needs to apply a region segmentation method to the input polarization data and to classify the region after segmentation. If the object 
is complicated and is rotated at a large angle, the class of the region will often change and will thus violate assumption 12. In addition, if the region changes in topology - for example, if it splits, vanishes, or regenerates then such a measurement also violates the assumption.

There are more interreflections in transparent objects than in opaque objects. According to our way of thinking, a method which can measure the shape of transparent objects is robustly applicable to any opaque objects. Thus, to prove the robustness of our method, we applied our method to transparent objects.

\subsection{Outline}

The structure of the remainder of this paper is as follows: In Section 2, we present a brief overview of the background theory of polarization[20] and show that one can determine surface orientation up to two possible incident angles by using the polarization. In Section 3, we describe the method for disambiguating the possibilities in the incident angle by rotating the object. In particular, we describe a method for determining the corresponding points; the method is based on the analysis of the Gauss map of the surface. In Section 4, we describe the apparatus used in this method and the experimental results. Finally, in Section 5, we conclude the paper.

\section{Polarization Analysis}

Light reflected from the surface of most types of objects can be separated into two major components: surface reflection and body reflection. Incident light partially reflects immediately from the surface and partially penetrates the object. The light that penetrates an opaque object randomly reflects at pigments inside the object and is emitted into the air. The light that immediately reflects into the air is called the surface reflection and the light that penetrates and is then reflected back into the air is called the body reflection[21]. Since, in this paper, we focus on transparent objects, we will deal only with surface reflection.

Generally, natural light is unpolarized and becomes polarized once it goes through a polarization material or it is reflected from a surface, and we are interested in measuring the degree of polarization of reflected light. The interfaces of smooth, transparent objects cause less diffuse reflection or absorption as opposed to those of opaque objects and the incident and reflecting angles are the same. Thus, once the reflecting angle and the orientation of the plane of incidence are known, we can determine the surface orientation 


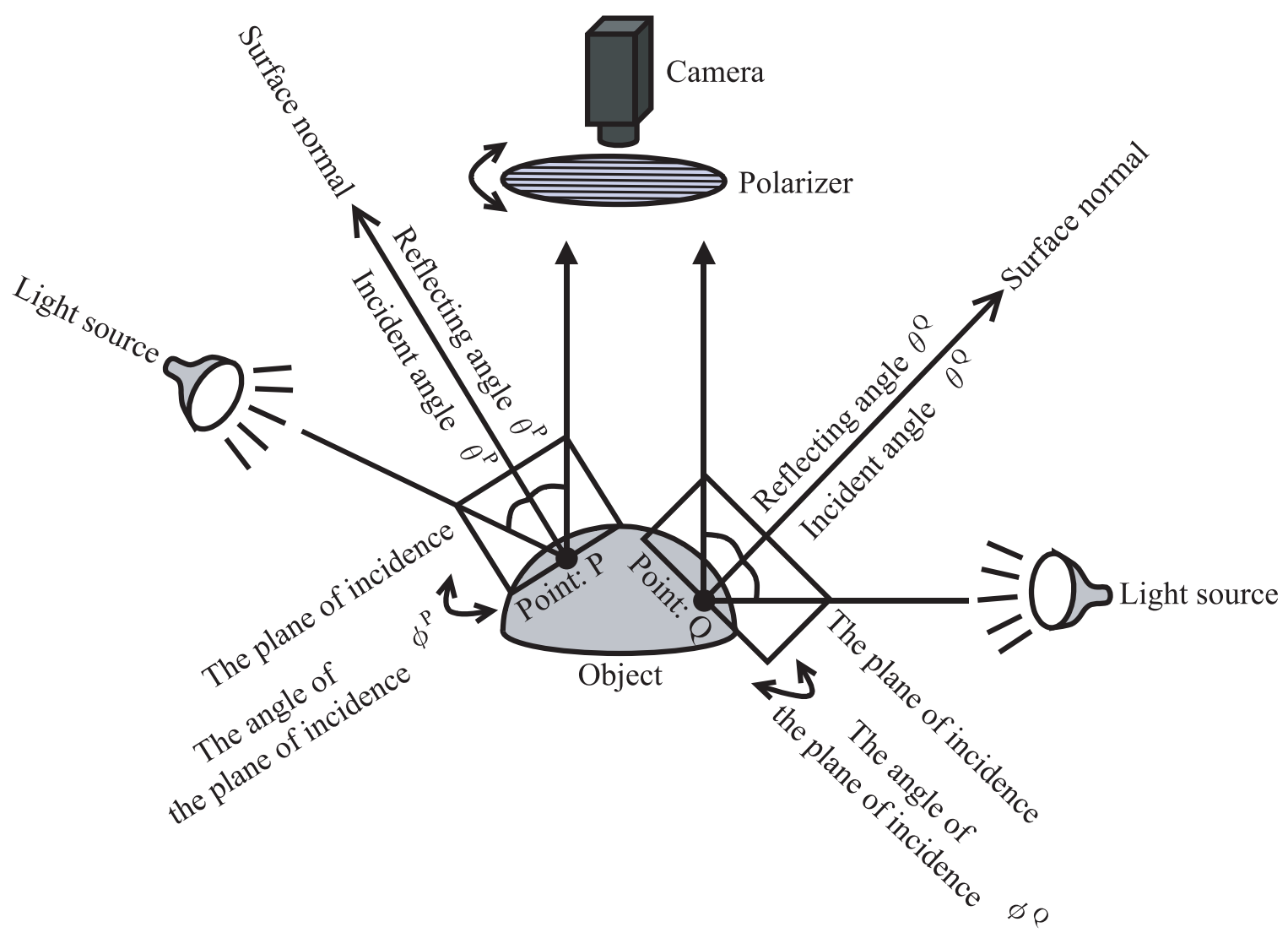

Figure 2: Surface normal of the object/

with respect to the viewer, as shown in Fig. 2. Here, the plane of incidence is the one on which the light source and surface normal lie. Since we analyze only smooth, transparent objects, the viewer vector is included in the plane of incidence. We will denote the direction of the plane of incidence and the reflecting angle as $\phi$ and $\theta$, respectively, and determine these two angles by using the degree of polarization of reflected light.

The reflectance ratio parallel to the plane of incidence, $F_{p}$, and the reflectance ratio perpendicular to the plane of incidence, $F_{s}$, are defined as:

$$
\begin{aligned}
& F_{p}=\frac{1+n^{2}-\left(n^{2}+1 / n^{2}\right) \sin ^{2} \theta-2 \cos \theta \sqrt{n^{2}-\sin ^{2} \theta}}{1+n^{2}-\left(n^{2}+1 / n^{2}\right) \sin ^{2} \theta+2 \cos \theta \sqrt{n^{2}-\sin ^{2} \theta}} \\
& F_{s}=\frac{1+n^{2}-2 \sin ^{2} \theta-2 \cos \theta \sqrt{n^{2}-\sin ^{2} \theta}}{1+n^{2}-2 \sin ^{2} \theta+2 \cos \theta \sqrt{n^{2}-\sin ^{2} \theta}},
\end{aligned}
$$

where $\theta$ is the incident angle and $n$ is the refractive index of the object relative to the air. The incident angle that satisfies $F_{p}=0$ is referred to as 
the Brewster angle, $\theta_{B}$. The Brewster angle is obtained as:

$$
\tan \theta_{B}=n
$$

\subsection{Direction of the Plane of Incidence, $\phi$}

As shown in (1), the intensity of the reflected light varies depending on the direction of oscillation in the plane of oscillation; therefore, a difference can be observed when the polarization filter is rotated in front of a CCD camera. The variance is described as a sinusoidal function of rotation angles. We will denote the maximum and minimum brightness in the observed intensities as $I_{\max }$ and $I_{\min }$. Given that the sum of the maximum and minimum brightness is the total brightness of the reflected light $I_{\text {spec }}$,

$$
I_{\max }=\frac{F_{s}}{F_{p}+F_{s}} I_{\text {spec }}, \quad I_{\min }=\frac{F_{p}}{F_{p}+F_{s}} I_{\text {spec }} .
$$

By this equation, the direction parallel to the plane of incidence provides the minimum brightness $I_{\min }$. Namely, by measuring the angle where the minimum brightness is observed, we can determine the direction of the plane of incidence $\phi(0 \leq \phi<2 \pi)$. There are two possible directions of the plane of incidence, $\phi_{\mathrm{LO}}$ and $\phi_{\mathrm{HI}}$, which are definable as $\phi_{\mathrm{HI}}=\phi_{\mathrm{LO}}+\pi$, where $0 \leq \phi_{\mathrm{LO}}<\pi$ and $\pi \leq \phi_{\mathrm{HI}}<2 \pi$.

Since we assume that the object is a closed, smooth object, we can determine the surface normal at the occluding boundary; the surface normal heads for the outside of the shape of the projection of the object at the occluding boundary. By using the $\phi$ at the occluding boundary as an initial condition, we propagate the constraint of $\phi$ throughout the surface and, finally, determine the value of $\phi$ over the entire surface, assuming that all local parts of the surface are not concave toward the camera direction.

\subsection{Incident Angle, $\theta$}

The definition of the degree of polarization (or polarization degree) is,

$$
\rho=\frac{I_{\max }-I_{\min }}{I_{\max }+I_{\min }}
$$

The degree of polarization is 0 when the light is un-polarized, whereas it is 1 when the light is linearly polarized. The linearly polarized light is observed when the incident angle and the reflecting angle are at the Brewster angle. 


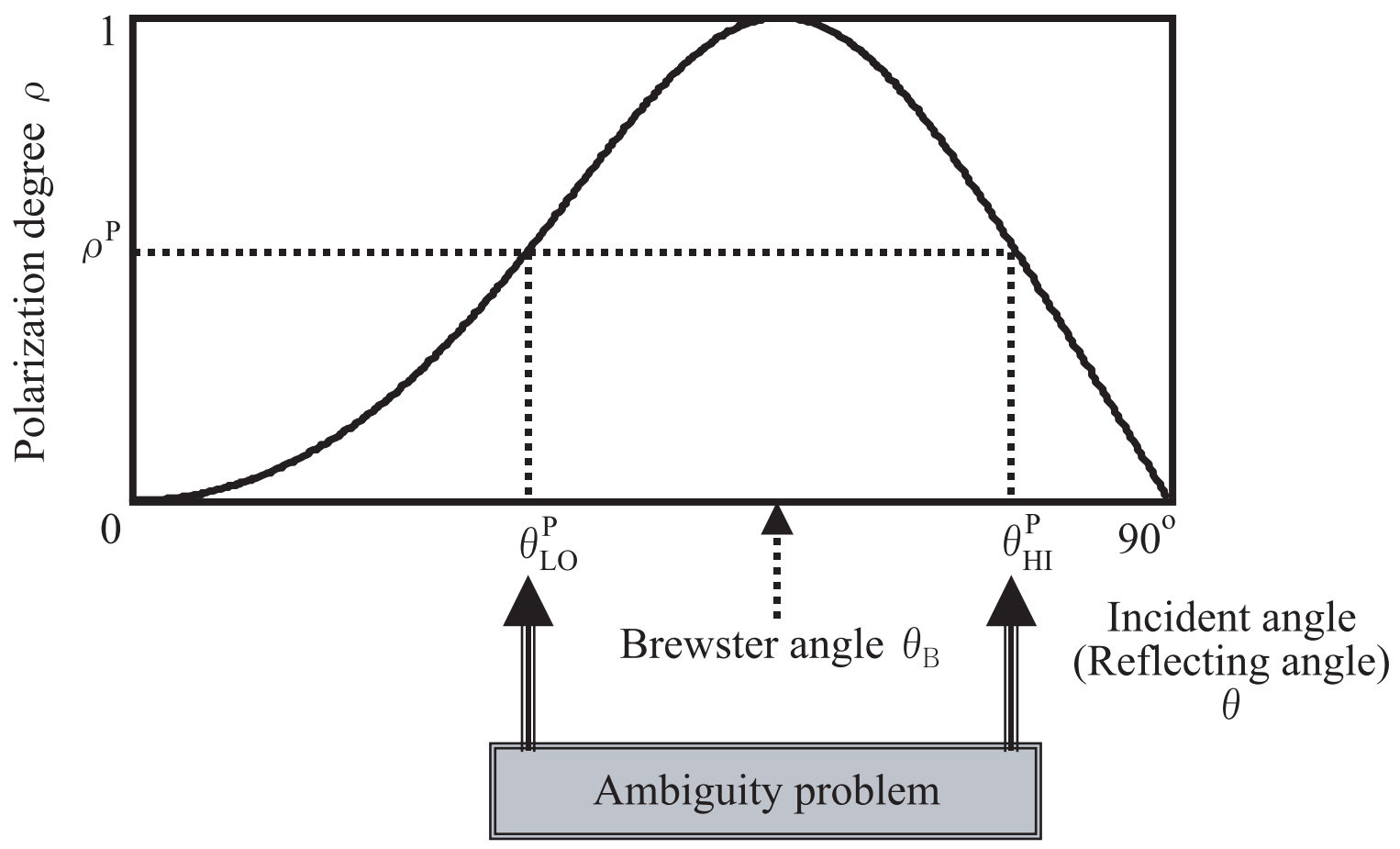

Figure 3: Relation between the degree of polarization and the incident angle $(n=1.5)$.

By substituting (3) and (1) into (4), we can represent the degree of polarization $\rho$ as

$$
\rho=\frac{2 \sin ^{2} \theta \sqrt{n^{2}-\sin ^{2} \theta-n^{2} \sin ^{2} \theta+\sin ^{4} \theta}}{n^{2}-\sin ^{2} \theta-n^{2} \sin ^{2} \theta+2 \sin ^{4} \theta} .
$$

The degree of polarization is a function of the refractive index $n$ and the incident angle $\theta(0 \leq \theta \leq \pi / 2)$. Thus, by obtaining the degree of polarization from the data, we can determine the incident angle $\theta$, given the refractive index $n$.

Fig. 3 shows the relationship between the degree of polarization and the incident angle. Here, the horizontal and vertical axes denote the incident angle and the degree of polarization, respectively. We can obtain the incident angle from the observed degree of polarization even if we do not know the intensity of the light source. The function has an extreme at the Brewster angle. From this function, an observed degree of polarization provides two possible incident angles, except at the Brewster angle. The method to resolve this ambiguity is described in the next section. 


\section{Disambiguation through Object Rotation}

Section 3 describes the method for solving the ambiguity problem and obtaining the surface normal of the object. We rotated the object to obtain two sets of polarization data from different viewing directions. First, we segmented each of the data of polarization degree into regions. Each region was classified into three types of regions as described in Section 3.1. Then, we detected corresponding points for each region and compared the value of degree of polarization at corresponding points. The corresponding points were detected by the method described in Section 3.2. Finally, Section 3.3 shows the method for solving the ambiguity problem by comparing the degree of polarization at corresponding points.

\subsection{Region Segmentation}

We have explained how to obtain the polarization degree of the light reflected on the object surface in Section 2. Now, we segment the data of polarization degree into some regions bounded by the Brewster angle $\theta_{B}$. Points of the Brewster angle have no ambiguity and the polarization degree $\rho$ is equal to 1. Since we assume that the object is a closed, smooth object, the curve connected by points of the Brewster angle will form a closed curve. This curve is sometimes thick, sometimes thin, and sometimes a combination of both. We denote a point where the zenith angle is equal to Brewster angle as the "Brewster point" and the closed curve consisting of Brewster points as the "Brewster curve." We define the segmentation by Brewster curves as "Brewster segmentation."

Now, let us consider the surface regions segmented with regard to the Brewster angle with a Gaussian sphere representation[1, 22]. The regions generated by Brewster segmentation can be grouped into three classes(Fig. 4):

1. B-E region - a region enclosed within a Brewster curve and an occluding boundary (mapped to the Equator on the Gaussian sphere),

2. B-N region - a region enclosed only with a Brewster curve and containing a surface orientation toward the viewer direction (mapped to the North Pole on the Gaussian sphere),

3. B-B region - a region enclosed only with one or more Brewster curve(s) and neither containing occluding boundary nor the surface normal facing the viewer. 


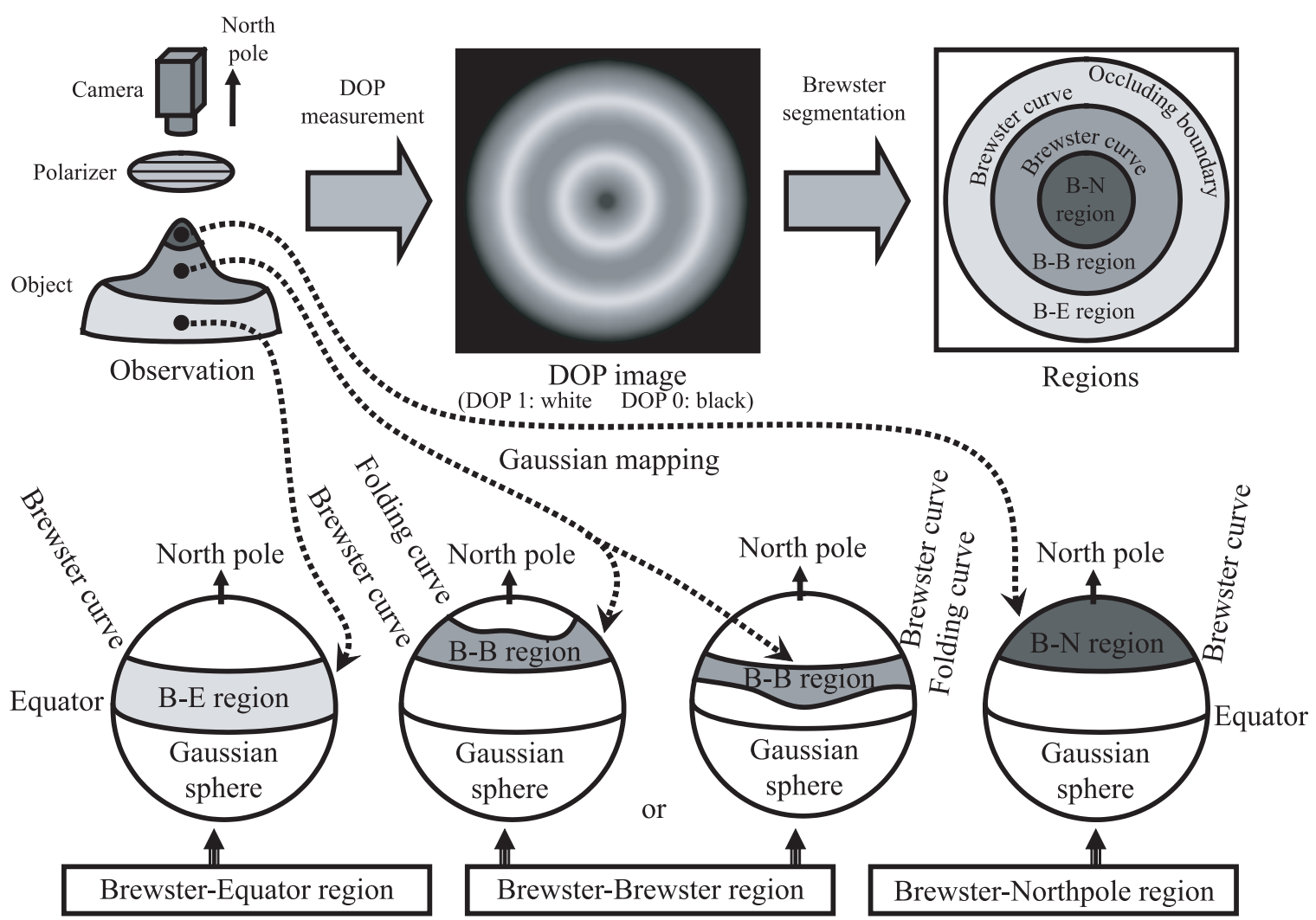

Figure 4: Gaussian mapping and regions.

' $B$ ' represents the capital letter $B$ of the Brewster curve, ' $E$ ' represents the capital letter $\mathrm{E}$ of the Equator, and ' $\mathrm{N}$ ' represents the capital letter $\mathrm{N}$ of the North Pole.

The result of the Brewster segmentation of the object depicted in Fig. 5 is shown in Fig. 6. Fig. 6a is a gray image of the polarization degree, where $\rho=0$ is represented as black and $\rho=1$ is represented as white. Fig. $6 \mathrm{~b}$ is the result of the Brewster segmentation of Fig. 6b. There are two Brewster curves and one occluding boundary and one each of B-E region, B-B region, and $\mathrm{B}-\mathrm{N}$ region.

The B-E region is the region which includes the occluding boundary whose zenith angle $\theta$ equals $90^{\circ}$. On the Gaussian sphere, B-E region is enclosed within a small circle mapped from the Brewster curve and an equator mapped from the occluding boundary. The zenith angle of all the points of B-E region is located between the Brewster angle and the occluding angle, $90^{\circ}$. The graph described in Fig. 3 indicates that the correspondence between $\theta$ and $\rho$ is one to one at this region, $\theta_{B} \leq \theta \leq 90^{\circ}$; thus, we can uniquely determine 


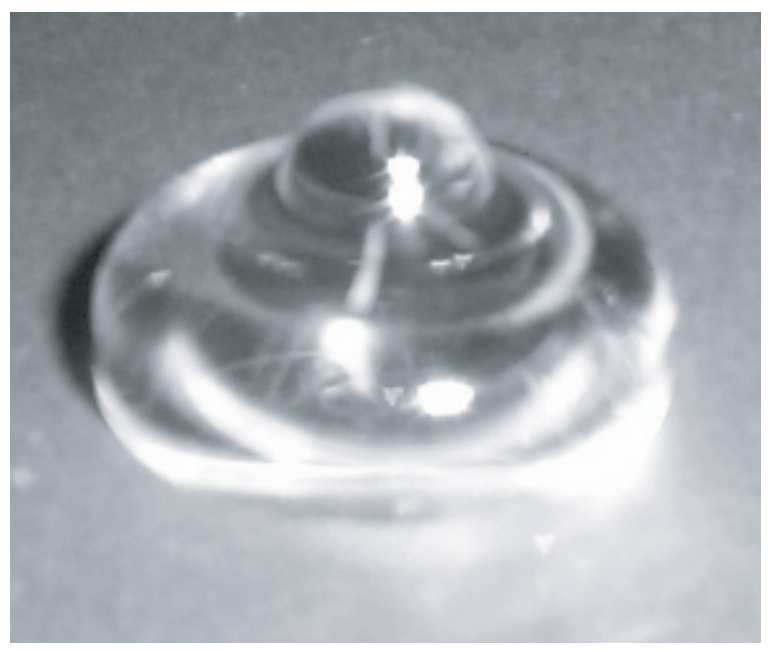

Figure 5: A photograph of the bell-shaped object.

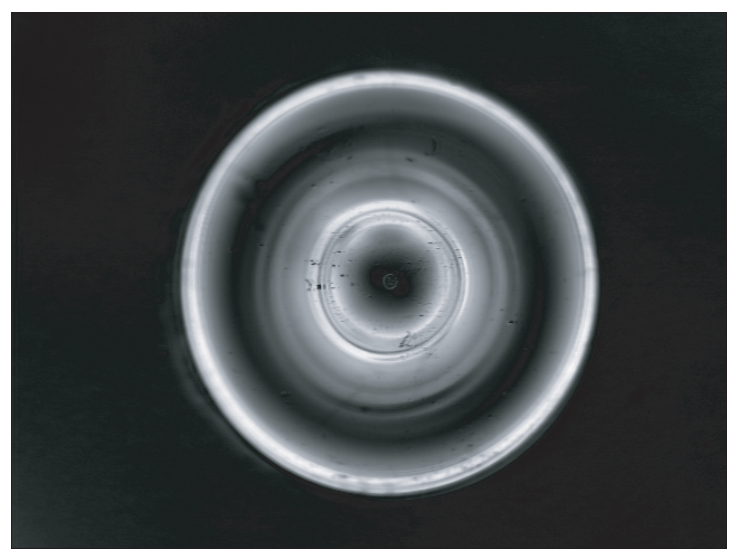

(a)

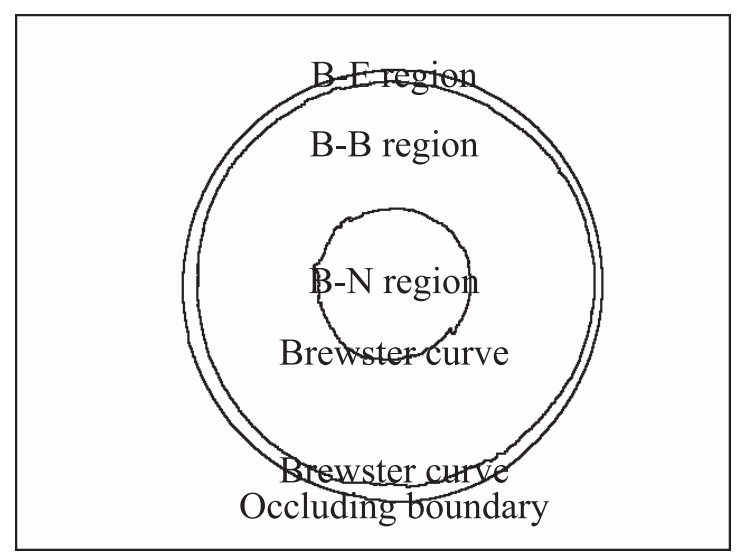

(b)

Figure 6: (a) A gray image of obtained polarization degree of the bell-shaped object and (b) the result of Brewster segmentation. 
the incident angle from an observed polarization degree, $\rho$.

The $\mathrm{B}-\mathrm{N}$ region is the region which includes the point(s) mapped onto the North Pole on the Gaussian sphere. As shown in Fig. 3, the region is mapped to a spherical cap on the Gaussian sphere, enclosed by a small circle mapped from the Brewster curve. The North Pole is located at the center of this spherical cap. The zenith angle of all the points in this region is in the range of $0^{\circ} \leq \theta \leq \theta_{B}$. From the graph in Fig. 3, we can also conclude that, in this range, the correspondence between $\theta$ and $\rho$ is one-to-one, and we can also determine the zenith angle from the observed polarization degree.

The B-B region is defined as the region which includes neither the occluding boundary nor the North Pole points and is bounded by one or more Brewster curves. In the following sections, we will propose a method for disambiguating $\mathrm{B}-\mathrm{B}$ regions.

\subsection{Corresponding Point}

There are two possibilities for the existence of the B-B region on the Gaussian sphere. The B-B region is either on the northern side of the Brewster curve or on the southern side of the Brewster curve. The B-B region mapped onto the Gaussian sphere is bounded by one Brewster curve and one or more extra curves. By considering the points in the B-B region on Gaussian sphere, we find that there is one extreme point - northernmost or southernmost - in each azimuth angle. We denote the set of these points to be a folding curve. Along this curve, the original surface is folded and is mapped two or more times between the folding curve and the Brewster curve.

Theorem Any folding curve on an object surface is a parabolic curve on that object surface. That is to say, at any surface point on a folding curve, the Gaussian curvature at the surface point vanishes.

The proof is provided in the Appendix. A parabolic curve is a curve where Gaussian curvature is zero and Gaussian curvature of object surface does not change through object rotation. Thus, we can conclude that the folding curve is intrinsic to an object and invariant from the viewer direction[22].

We obtain one set of data of the polarization degree for input data. However, one set of data is not enough for resolving the ambiguity in the B-B region; thus, we have to obtain extra data: we tilt(rotate) the object at a small angle and obtain additional data of polarization degrees (Fig. 7). We find identical points(corresponding points or matching points) of those two sets of data and compare the polarization degrees of two data at identical points in order to solve the ambiguity in the B-B region. 


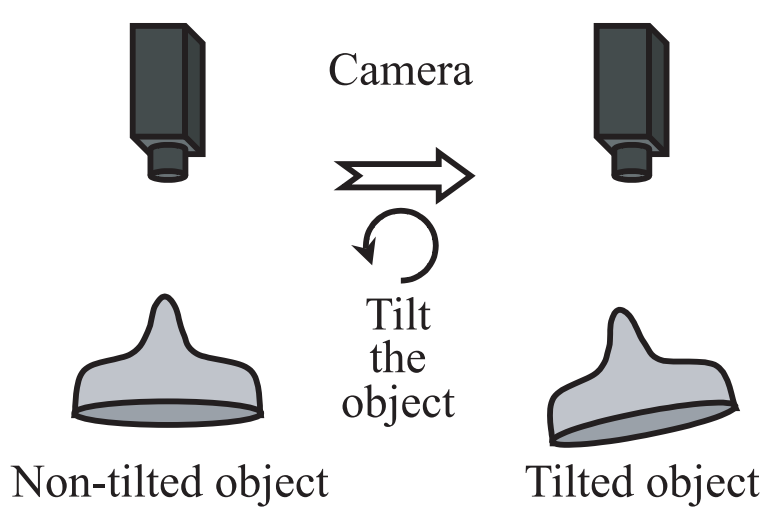

Figure 7: Object rotation.

When rotating the object, images mapped onto the Gaussian sphere are also rotated in a similar manner. Since the folding curve is intrinsic to the object, the folding curve also rotates in the same manner. On the other hand, the Brewster curve depends on both surface normals and viewer direction, so it is not invariant through the object rotation. As a result, we can use the folding curve for the matching. The intersection of the folding curve and the great circle, which represents the rotation direction, is defined as the corresponding point(Fig. 8). This great circle must be a cross-section between the Gaussian sphere and the plane which is parallel to the rotation direction of the object and includes the two poles of the Gaussian sphere. The surface point which is mapped onto this great circle still exists in this great circle after the object rotation, thereby making unique matching possible.

If the $\mathrm{B}-\mathrm{B}$ region is mapped onto the northern side of the Brewster curve, we can choose the northernmost point for the corresponding point which intersects the great circle, namely, we use the point where the polarization degree is minimum. If the B-B region is mapped onto the southern side of the Brewster curve, we can choose the nearest point to the equator for the corresponding point which intersects the great circle, namely, we use the point where the polarization degree is minimum.

Our conclusion is that the point of the $\mathrm{B}-\mathrm{B}$ region where the polarization degree is minimum and the surface normal lies along the rotation direction is the best corresponding point to adopt.

\subsection{Difference of Polarization Degree}

Finally, we describe the method used to resolve the ambiguity problem of the surface normal by comparing the polarization degree at the corresponding 


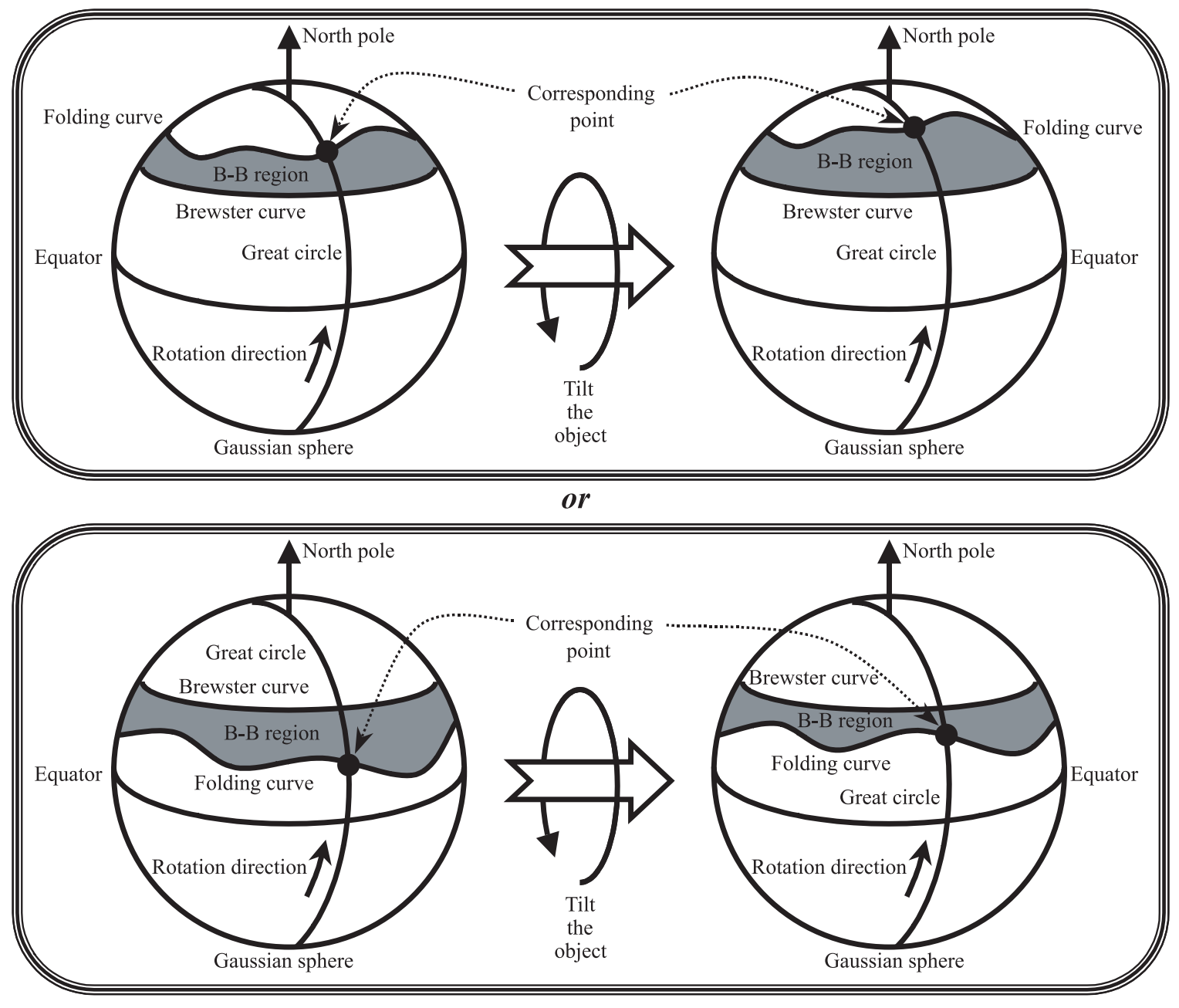

Figure 8: Corresponding Point. 
point of the nontilted object with that of the tilted object.

We regard the refractive index $n$ as constant, making the polarization degree $\rho$ a function of only the zenith angle $\theta$. The relationship between the rotation angle, $\Delta \theta$, the polarization degree of the nontilted object, $\rho(\theta)$, the polarization degree of the tilted object, $\rho(\theta+\Delta \theta)$, and the derivative of the polarization degree, $\rho^{\prime}(\theta)$, will be:

$$
\operatorname{sgn}\left(\rho^{\prime}(\theta)\right)=\frac{\operatorname{sgn}(\rho(\theta+\Delta \theta)-\rho(\theta))}{\operatorname{sgn}(\Delta \theta)},
$$

where $\operatorname{sgn}(x)$ is a function which returns the $\operatorname{sign}$ of $x$. Note that we assume that the rotation angle $\Delta \theta$ is sufficiently small.

The derivative of the polarization degree $\rho$ by the zenith angle $\theta$ is:

$$
\frac{d \rho}{d \theta}=\frac{2 \sin \theta\left(n^{2}-\sin ^{2} \theta-n^{2} \sin ^{2} \theta\right)\left(2 n^{2}-\sin ^{2} \theta-n^{2} \sin ^{2} \theta\right)}{\sqrt{n^{2}-\sin ^{2} \theta}\left(n^{2}-\sin ^{2} \theta-n^{2} \sin ^{2} \theta+2 \sin ^{4} \theta\right)^{2}} .
$$

The graph of the polarization degree is depicted in the lower half of Fig. 9 and the graph of the derivative of the polarization degree is depicted in the upper half of Fig. 9. The derivative of the polarization degree $d \rho / d \theta$ is positive when $0<\theta<\theta_{B}$ and is negative when $\theta_{B}<\theta \leq \pi / 2$.

In fact, we do not need to know the absolute value of the rotation angle; however, we assume that we know the rotation direction. Since the azimuth angle $\phi$ has also already been determined, we can determine the sign of $\Delta \theta$. As a result, by calculating the sign of the difference of two polarization degrees at the corresponding point and by giving the sign of $\Delta \theta$, we can determine, by using $(6)$, whether the zenith angle $\theta$ in $\mathrm{B}-\mathrm{B}$ region is in the range of $0 \leq \theta \leq \theta_{B}$ or of $\theta_{B} \leq \theta \leq \pi / 2$.

The correspondence between the polarization degree $\rho$ and the zenith angle $\theta$ is one-to-one in the range of $0 \leq \theta \leq \theta_{B}$, and is also one-to-one in the range of $\theta_{B} \leq \theta \leq \pi / 2$. Therefore, if we simply determine that the zenith angle $\theta$ in $\mathrm{B}-\mathrm{B}$ region is in the range of whether $0 \leq \theta \leq \theta_{B}$ or $\theta_{B} \leq \theta \leq \pi / 2$ by (6), we can determine the zenith angle $\theta$ uniquely from the value of the polarization degree $\rho$.

\section{Experiments}

\subsection{Experimental Setup}

Fig. 10 shows the apparatus for the measurement. As a light source, we use a spherical diffuser illuminated from three $300 \mathrm{~W}$ incandescent light bulbs, 


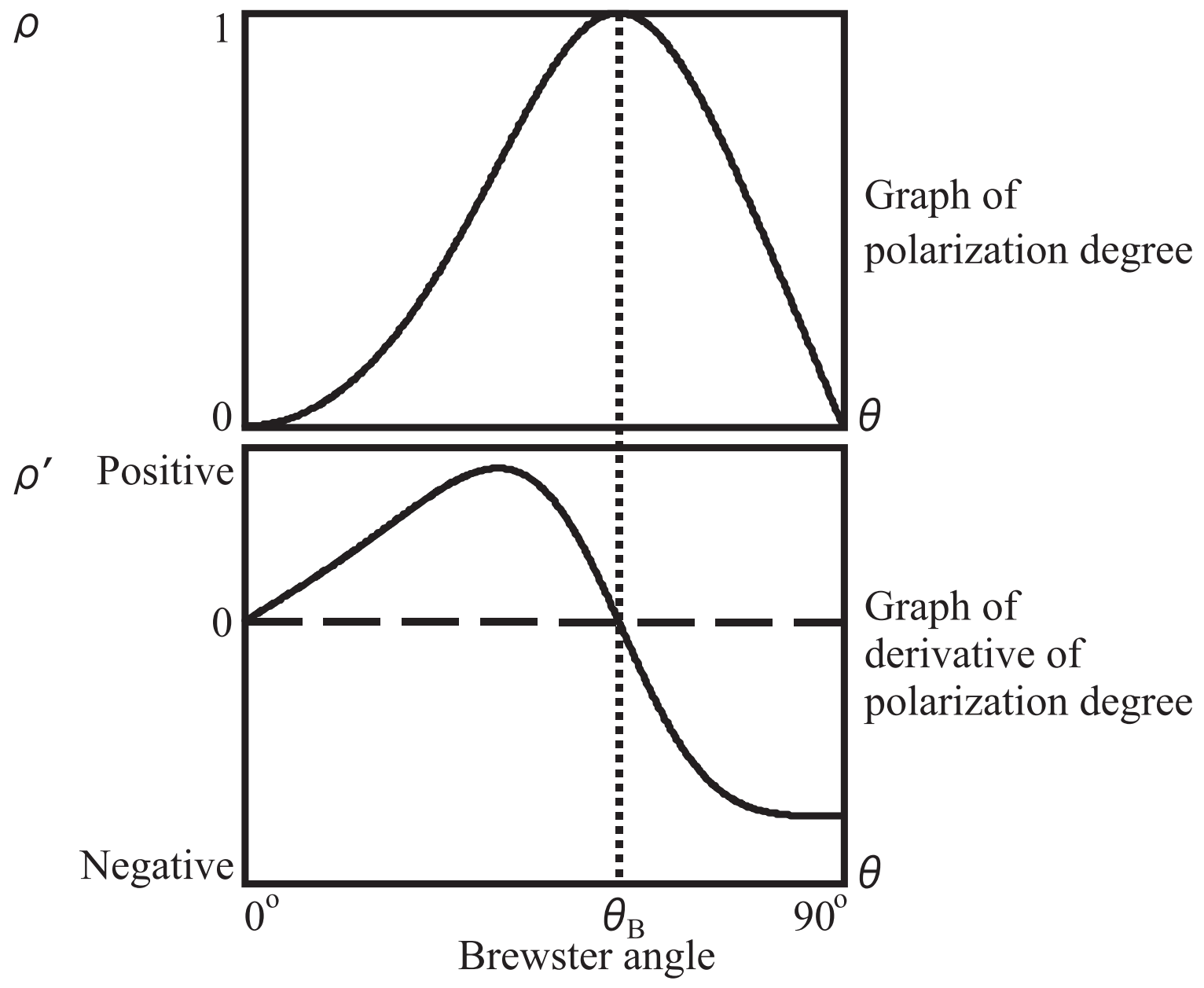

Figure 9: Graph of derivative of polarization degree $(n=1.5)$. 


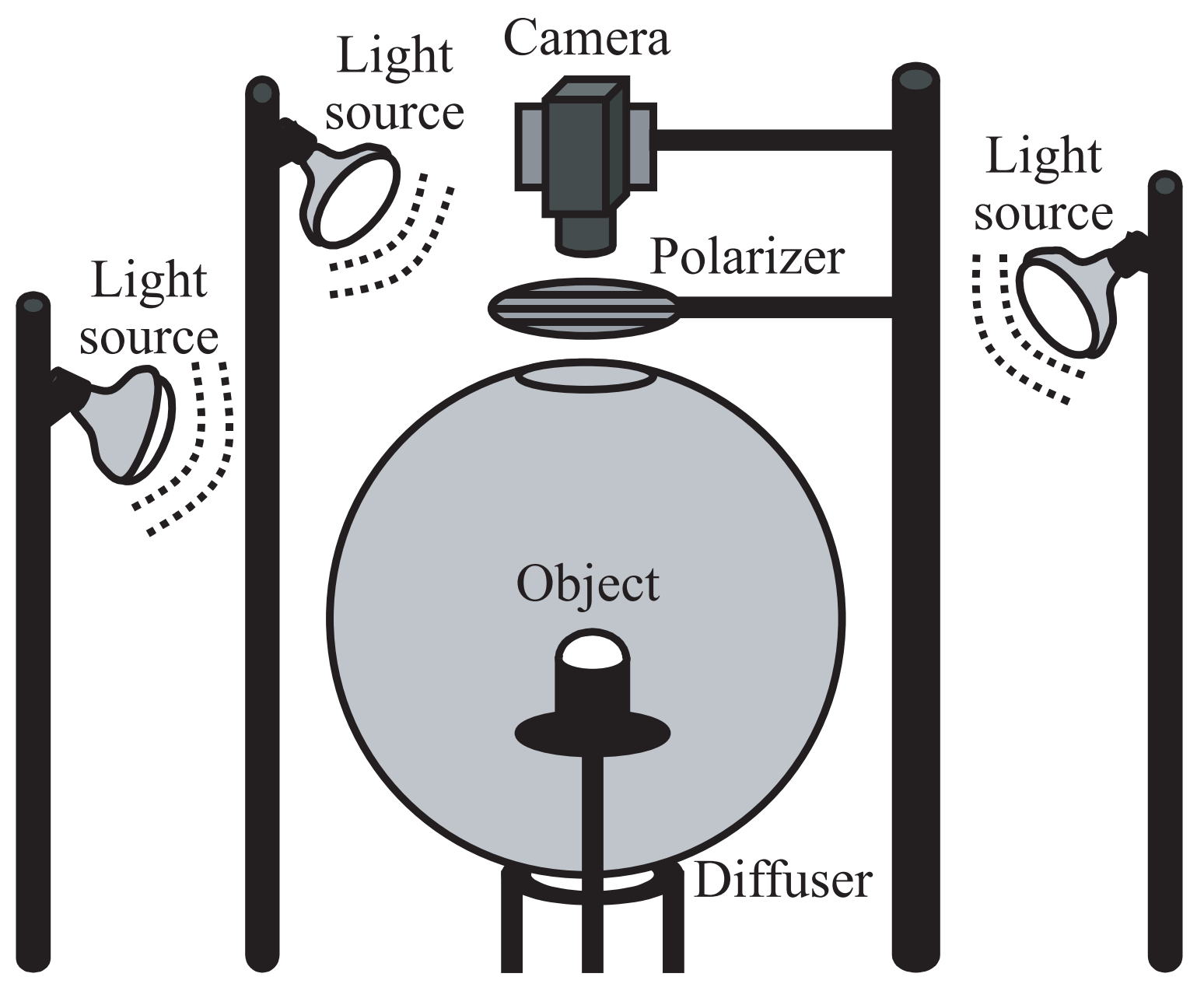

Figure 10: Experimental setup.

located circularly at 120 degrees apart. The spherical diffuser is made of plastic, and its diameter is $40 \mathrm{~cm}$. This spherical diffuser becomes an unpolarized spherical light source and illuminates an object that is located at the center of the sphere from all directions. Because we determine surface orientations using only surface reflection and the surface reflection occurs only when the reflecting and incident angles are the same, it is necessary to illuminate an object from all directions in order to observe surface reflections over the entire object surface. The object is observed through a small hole at the top of the sphere by a monochrome CCD camera. A polarization filter is mounted between the hole and the camera.

When the light of an incandescent lamp penetrates the white plastic diffuser, the light will be unpolarized while randomly scattered inside the dif- 
fuser. The distance between the diffuser and the object is large enough compared with the size of the object, thus the observed light reflected at the object surface originally comes from the inner surface of the diffuser with same emitting direction as the surface normal of the diffuser. For this emitting angle, the light does not polarize[20]. Thus, this spherical diffuser provides an unpolarized light.

\subsection{Measurement Procedures}

A transparent object reflects and transmits light in a complex manner causing multiple interreflections which interfere with our observations. We set the object on a black pipe to block the light coming from behind the object; however, some interreflection still occurs. The interreflection lowers the degree of polarization. To overcome it, we simply subtract an unpolarized light from the input data and increase the degree of polarization. Since we do not know the information of the interreflection, we simply assume that the unpolarized and uniform light caused by interreflection is added with the light which is directly reflected from the object surface only once. This assumption is not always true for each point on object surface, but we expect that this assumption statistically holds for sets of many points on the object surface. We assume that the object is a closed, smooth object; thus, the Brewster angle will always appear. Thus, the maximum value of the degree of polarization must be "1." We choose a certain number of points where the degree of polarization is high, and estimate the subtraction value as the average value which makes the degree of polarization of each chosen point be "1." This modification raises the maximum polarization degree of raw data to 1 . The modified polarization degree $\rho^{\prime}$ is calculated by the following equation which is derived by modifying (4):

$$
\rho^{\prime}=\frac{I_{\max }-I_{\min }}{I_{\max }+I_{\min }-u} .
$$

where $u / 2$ is the intensity of the estimated unpolarized light. $u$ is estimated by setting $\rho^{\prime}$ as 1 for the chosen surface points.

By rotating a polarization filter, we obtain a sequence of images of an object. We measure from 0 to 175 degrees at 5 degree intervals. From this process, we obtain 36 images. We observe variance of intensity at each pixel of the 36 images. By using the least-squares minimization, we fit a sinusoidal curve to those intensities and then determine the maximum and minimum intensities, $I_{\max }$ and $I_{\min }$. From those values, we determine two possible surface orientations by using the algorithm. 
After applying this measurement to the nontilted object, we apply the same measurement to the tilted object and obtain the polarization degree of this second view. A proper rotation direction is provided by a human operator because, on the Gaussian sphere, the great circle must intersect $\mathrm{B}-\mathrm{B}$ region to solve the ambiguity problem.

We applied the "region growing" method[23] for Brewster segmentation. Initial values for the "region growing" method are given by the human operator. Since the "1" polarization degree cannot be observed in any cases, we therefore segment the regions by a closed curve of the maximum polarization degree. We call a curve of the maximum polarization degree a pseudoBrewster curve. A folding curve also exists in the pseudo-B-B region - a region bounded by pseudo-Brewster curve. Consequently, the segmentation is still useful not only for the Brewster curve, but also for the pseudo-Brewster curve.

We compare those two sets of data at each corresponding point. To determine the corresponding points of the two sets of data, we first classify the regions generated by separating the polarization data with regard to the Brewster angle into three classes: the $\mathrm{B}-\mathrm{E}$ region, the $\mathrm{B}-\mathrm{N}$ region, and the $\mathrm{B}-\mathrm{B}$ region. Then, we detect a minimum value of the polarization degree in each B-B region whose surface normal has the same orientation as the rotating direction. Finally, the difference value of the polarization degrees in those two corresponding points solves the ambiguity problem of the angle; as a result, we obtain the correct incident angle of the object surface.

\subsection{Measurement Results}

First, we analyzed the precision of the measurement system before applying our proposed method. For this experiment, we used an acrylic transparent hemisphere whose refractive index was 1.5 and diameter was $30 \mathrm{~mm}$. The refractive index was obtained from the literature[24]. Since we knew that the shape was a hemisphere, the computed data became comparable with the ground truth. Error was calculated as an average value throughout the entire object surface, i.e., computed as an absolute difference between the true value and the obtained value. The errors of polarization degree, incident angle, and height were $0.17,8.5^{\circ}$, and $1.1 \mathrm{~mm}$, respectively. Since the radius of the hemisphere was $15 \mathrm{~mm}$, the true average height was $10 \mathrm{~mm}$. Therefore, the error rate,

$$
\frac{\text { average of |true height }- \text { calculated height } \mid}{\text { average of true height }}
$$

was 11 percent for this measurement. 


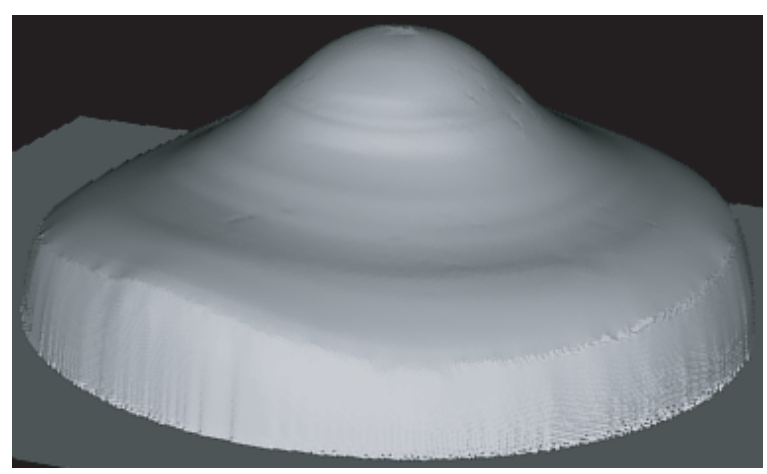

Figure 11: A rendered image of the obtained shape of the bell-shaped object.

We also measured the heart-shaped piece of glass depicted in Fig. 1a. There was no B-B region, making the object rotation unnecessary. The obtained shape is shown in Fig. $1 \mathrm{~b}$.

In order to demonstrate the applicability of our system to a real object of more general shape than a hemisphere, we determined the shape of the bell-shaped object shown in Fig. 5. The object was made of acrylic and its refractive index is 1.5 , obtained from the literature[24]. We tilted the object approximately 8 degrees and obtained the data from two views. By applying our method to the data that had been obtained, we calculated the distribution of the surface normal of the object. Then, we used a relaxation algorithm $[1,25]$ to convert the orientation distribution into a shape corresponding to that of the object. Fig. 11 shows the rendered image of the estimated shape of the object. Fig. 12 illustrates how the estimated shape fitted the true shape. Dots represent the obtained height and a solid line represents the ground truth, which was obtained by hand using the edge from the photo of the object observed from the side. The diameter(width) of the object was $24 \mathrm{~mm}$ and the height was $8 \mathrm{~mm}$. An average error(=absolute difference) of the height was $0.4 \mathrm{~mm}$.

A small error exists in the estimated shape, probably due to the interreflection of the object. Interreflection lowers the degree of polarization. This means that the zenith angle smaller than Brewster angle becomes smaller and the angle larger than Brewster angle becomes larger. However, the observed area of the smaller zenith angle is larger than that of larger angle. Thus, the resulting height will often be lower than the ground truth; the difference of the height is squeezed. We modify the input data and raise the degree of polarization; however, the modification is not ideal; thus, the resultant height will be different from the truth value. 


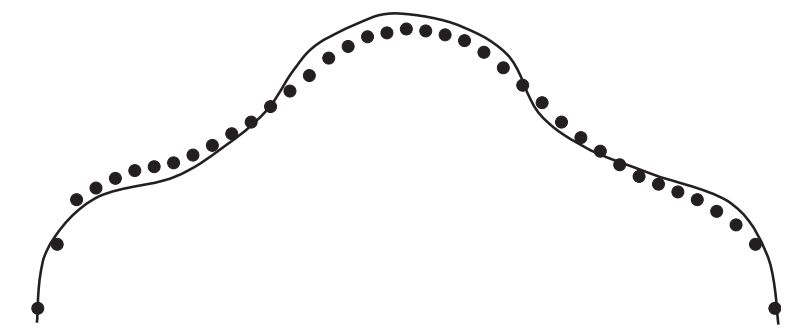

Figure 12: The result of the real bell-shaped object.

Another transparent object shown in Fig. 13a was measured. This moutain-shaped object was made of epoxy and its refractive index was 1.6[24]. The diameter(width) of the object was $45 \mathrm{~mm}$ and the height was $25 \mathrm{~mm}$. Fig. $13 \mathrm{~b}$ shows the result of region segmentation. Here, one B-E region, one B-N region, and four $\mathrm{B}-\mathrm{B}$ regions are observed. We rotated the object approximately 8 degrees. Figs. $13 \mathrm{c}$ and $13 \mathrm{~d}$ represent the estimated shape of the object.

\section{Conclusions}

In this paper, we have proposed a method for determining the shape of a transparent object by using polarization filter. Surface orientations are determined by using the polarization data. Because an algorithm that uses only one view results in ambiguities, polarization of a slightly tilted view is also employed.

We obtain two sets of data: One is from the object not tilted, and the other is from the object tilted at a small angle. We segment these data into some regions with regard to the Brewster angle. We calculate the difference of the polarization degree between these two sets of data at the corresponding point - the point where surface normal lies along the rotation direction and where the polarization degree is minimum in the B-B region. From that difference, we determine the correct surface normal.

We have implemented the proposed method and demonstrated its ability to determine the shape of real transparent objects. First, we analyzed the precision of the measurement system by measuring a transparent hemisphere. Then, we demonstrated the ability of the system to determine the shapes of transparent objects whose shapes are more complex than those of spherical objects.

The proposed method solves the ambiguity problem, and determines the shape of transparent objects more easily than do the methods in previous 


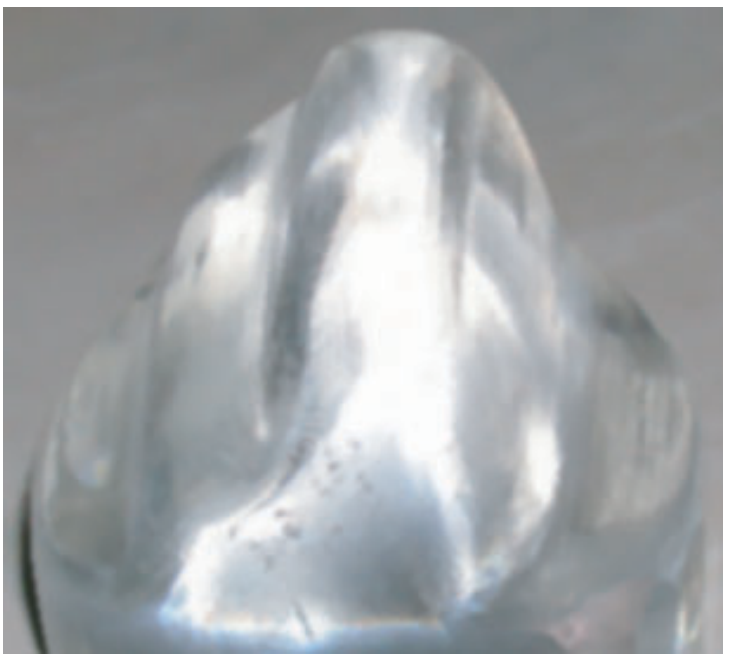

(a)

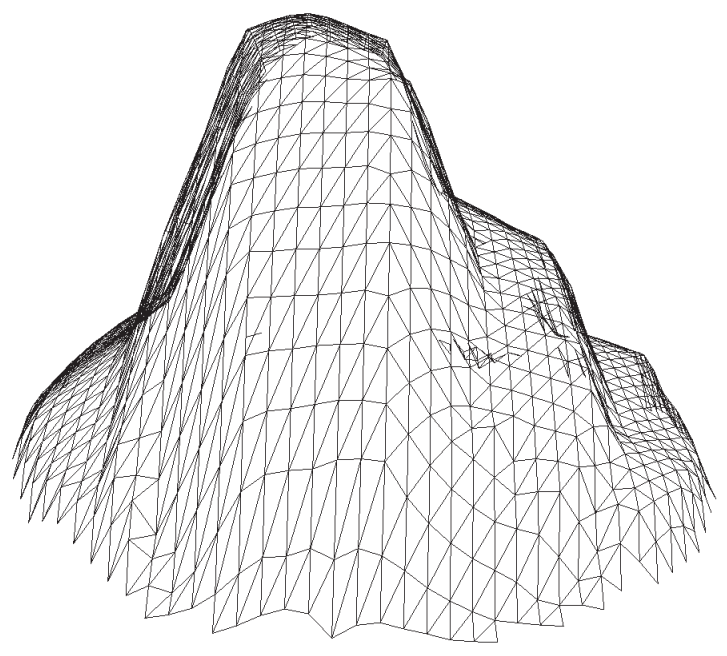

(c)

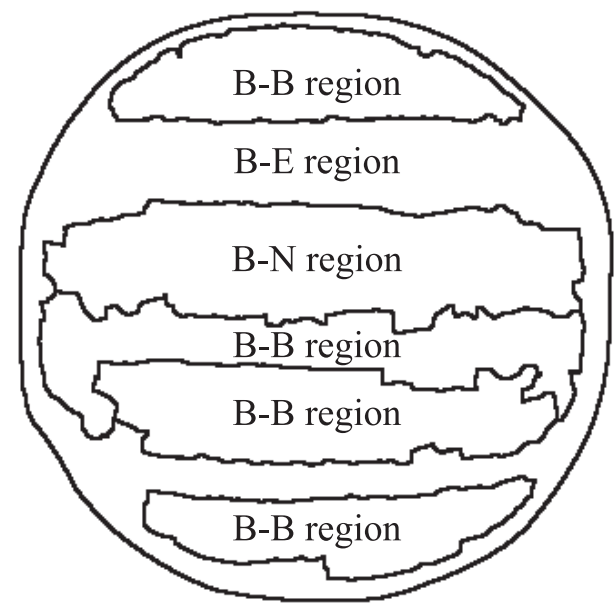

(b)

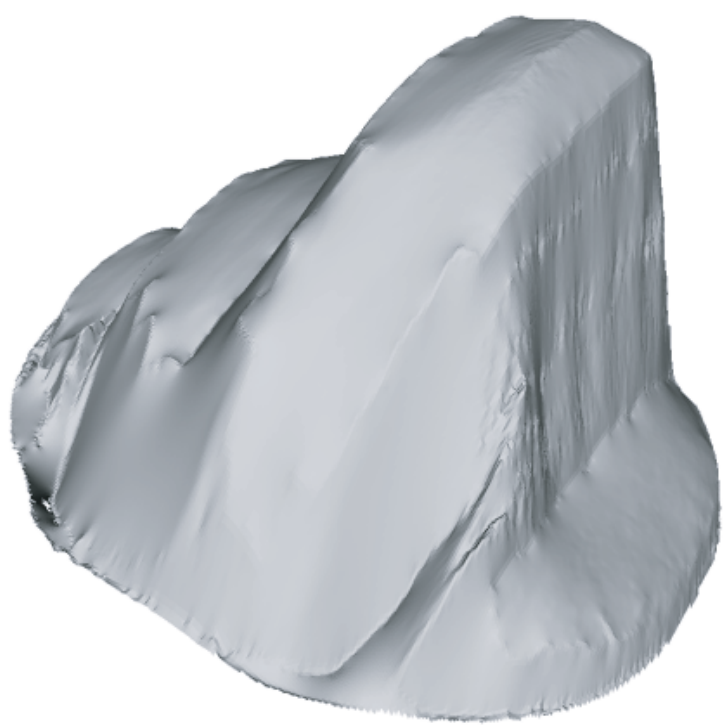

(d)

Figure 13: Measurement result of transparent moutain-shaped object: (a) Real image, (b) region segmentation result, and (c) and (d) rendered image. 
work. The proposed method works robustly for transparent objects which have less interreflection. Our method can obtain the shape of a closed, smooth transparent object with no deep-concave parts. Our future work is to develop a method that can handle the influence of interreflection.

The application field of the modeling of transparent objects can range from computer-aided manufacturing, classifying garbage/rubbish for recycling glass and plastic bottles, modeling cultural assets, to creating $3 \mathrm{D}$ catalogs for online shopping, etc. For the first step for such a wide area of applications, we proposed a basic technique for modeling the surface shape of transparent objects.

\section{A Appendix: Parabolic Curve}

Theorem Any folding curve is a parabolic curve on an object surface. That is to say, at any point on a folding curve, the Gaussian curvature at the point vanishes.

Proof. A surface normal can be represented in gradient space, a space constructed by gradients $p$ and $q$ :

$$
p=\frac{\partial H}{\partial x}, \quad q=\frac{\partial H}{\partial y}
$$

where $H=H(x, y)$ denotes the height of the object surface. A folding curve is an extremum not only in a Gaussian sphere, but also in gradient space, $p=p(x, y)$ and $q=q(x, y)$. Thus, one or both of the following equation holds:

$$
\begin{aligned}
& \frac{\partial p}{\partial x}=\frac{\partial p}{\partial y}=0 \\
& \frac{\partial q}{\partial x}=\frac{\partial q}{\partial y}=0
\end{aligned}
$$

Hessian $\mathcal{H}$ and Gaussian curvature $K$ are related by the following equation[22]:

$$
\operatorname{sgn} K=\operatorname{sgn} \operatorname{det} \mathcal{H}
$$

where Hessian is defined as:

$$
\mathcal{H}=\left(\begin{array}{ll}
\frac{\partial^{2} H}{\partial x^{2}} & \frac{\partial^{2} H}{\partial x \partial y} \\
\frac{\partial^{2} H}{\partial y \partial x} & \frac{\partial^{2} H}{\partial y^{2}}
\end{array}\right)
$$

Since (11) or (12) holds, from (10)-(14), we finally obtain $K=0$. 


\section{Acknowledgments}

This research was supported in part by the Japan Science and Technology Agency under the Ikeuchi CREST project. This research was conducted through cooperation in part with Megumi Saito, Yoichi Sato, and Hiroshi Kashiwagi. Polarizers were provided/developed by Polatechno Co., Ltd., Furuuchi Chemical Corporation, etc. The authors thank Ko Nishino, Robby T. Tan, and Marie Elm for proofreading and editing this manuscript. They also thank David Jacobs and the anonymous referees for their careful reviews of the paper.

\section{References}

[1] B.K.P. Horn, Robot Vision, p. 509. Cambridge, Mass.: MIT Press, 1986.

[2] D.H. Ballard and C.M. Brown, Computer Vision, p. 523. Englewood Cliffs, N.J.: Prentice Hall, 1982.

[3] K. Ikeuchi, "Determining Surface Orientations of Specular Surfaces by Using the Photometric Stereo Method," IEEE Trans. Pattern Analysis and Machine Intelligence, vol. 3, no. 6, pp. 661-669, 1981.

[4] S.K. Nayar, K. Ikeuchi, and T. Kanade, "Determining Shape and Reflectance of Hybrid Surface by Photometric Sampling," IEEE Trans. Robotics and Automation, vol. 6, no. 4, pp. 418-431, Aug. 1990.

[5] Y. Sato and K. Ikeuchi, "Temporal-Color Space Analysis of Reflection," $J$. Optical Soc. Am. A, vol. 11, no. 11, pp. 2990-3002, Nov. 1994.

[6] M. Oren and S.K. Nayar, "A Theory of Specular Surface Geometry," Int'l J. Computer Vision, vol. 24, no. 2, pp. 105-124, Sept. 1997.

[7] K. Koshikawa, "A Polarimetric Approach to Shape Understanding of Glossy Objects," Proc. Int'l Joint Conf. Artificial Intelligence, pp.493-495, 1979.

[8] K. Koshikawa and Y. Shirai, "A Model-Based Recognition of Glossy Objects Using Their Polarimetrical Properties," Advances in Robotics, vol. 2, no. 2, pp. 137-147, 1987.

[9] L.B. Wolff, "Polarization-Based Material Classification from Specular Reflection," IEEE Trans. Pattern Analysis and Machine Intelligence, vol. 12, no. 11, pp. 1059-1071, Nov. 1990. 
[10] L.B. Wolff and T.E. Boult, "Constraining Object Features Using a Polarization Reflectance Model," IEEE Trans. Pattern Analysis and Machine Intelligence, vol. 13, no. 7, pp. 635-657, July 1991.

[11] S. Rahmann and N. Canterakis, "Reconstruction of Specular Surfaces Using Polarization Imaging," Proc. IEEE Conf. Computer Vision and Pattern Recognition, pp.149-155, 2001.

[12] R. Szeliski, S. Avidan, and P. Anandan, "Layer Extraction from Multiple Images Containing Reflections and Transparency," Proc. IEEE Conf. Computer Vision and Pattern Recognition, pp.246-253, 2000.

[13] Y. Schechner, J. Shamir, and N. Kiryati, "Polarization-Based Decorrelation of Transparent Layers: The Inclination Angle of an Invisible Surface," Proc. IEEE Int'l Conf. Computer Vision, pp.814-819, 1999.

[14] D.E. Zongker, D.M. Warner, B. Curless, and D.H. Salesin, "Environmental Matting and Compositing," Proc. SIGGRAPH, pp.205-214, 1999.

[15] Y. Chuang, D.E. Zongker, J. Hindorff, B. Curless, D.H. Salesin, and R. Szeliski, "Environment Matting Extensions: Towards Higher Accuracy and Real-Time Capture," Proc. SIGGRAPH, pp.121-130, 2000.

[16] Y. Wexler, A. Fitzgibbon, and A. Zisserman, "Image-Based Environment Matting," Proc. Eurographics Workshop Rendering, pp.289-299, 2002.

[17] W. Matusik, H. Pfister, R. Ziegler, A. Ngan, and L. McMillan, “Acquisition and Rendering of Transparet and Refractive Objects," Proc. Eurographics Workshop Rendering, pp.267-278, 2002.

[18] M. Saito, Y. Sato, K. Ikeuchi, and H. Kashiwagi, "Measurement of Surface Orientations of Transparent Objects by Use of Polarization in Highlight," $J$. Opt. Soc. Am. A, vol. 16, no. 9, pp. 2286-2293, Sept. 1999.

[19] D. Miyazaki, M. Saito, Y. Sato, and K. Ikeuchi, "Determining Surface Orientations of Transparent Objects Based on Polarization Degrees in Visible and Infrared Wavelength," J. Opt. Soc. Am. A, vol. 19, no. 4, pp. 687-694, Apr. 2002 .

[20] M. Born and E. Wolf, Principles of Optics, p. 803. London: Pergamon Press, 1959 .

[21] K.E. Torrance and E.M. Sparrow, "Theory for Off-Specular Reflection from Roughened Surfaces," J. Opt. Soc. Am., vol. 57, no. 9, pp. 1105-1114, Sept. 1967 . 
[22] M.P. do Carmo, Differential Geometry of Curves and Surfaces, p. 503. Englewood Cliffs, N.J.: Prentice Hall, 1976.

[23] R.C. Gonzalez and R.E. Woods, Digital Image Processing, p. 716. Reading, Mass.: Addison Wesley, 1993.

[24] J.F. Shackelford, W. Alexander, and J.S. Park, CRC Materials Science and Engineering Handbook, p. 883. Boca Raton, Fla.: CRC Press, 1994.

[25] K. Ikeuchi, "Reconstructing a Depth Map from Intensity Maps," Proc. Int'l Conf. Pattern Recognition, pp.736-738, 1984.

Daisuke Miyazaki received the BS degree in science from the University of Tokyo in 2000, and the MS degree in information science and technology from the University of Tokyo in 2002. He is a $\mathrm{PhD}$ student at the Graduate School of Information Science and Technology at the University of Tokyo, Japan. He received the Best Overall Paper Award from VSMM in 2000. His research interests include physics-based vision and image-based modeling.

Masataka Kagesawa recieved the BS degree in mathematics in 1986 from Chiba University, Japan, and the MS degree in mathematics from Tokyo Metropolitan University in 1988. He was a doctoral course student at Tokyo Metropolitan University from 1988 to 1990. From 1990 to 1994, he was a technical associate at the Institute of Industrial Science, the University of Tokyo. He is now a research associate at the same institute. His research interests include traffic simulation with dynamic information, traffic management systems, and sensing systems for intelligent road traffic systems.

Katsushi Ikeuchi (F'98) received the $\mathrm{PhD}$ degree in information engineering from the University of Tokyo, Japan, in 1978. He is a professor at the Institute of Industrial Science, the University of Tokyo, Tokyo, Japan. After working at the AI Laboratory of MIT for three years, the ETL for five years, and the School of Computer Science of CMU for 10 years, he joined 
the university in 1996. Dr. Ikeuchi has served as the program/general chairman of several international conferences, including 1995 IEEE-IROS, 1996 IEEE-CVPR, and 1999 IEEE-ITSC. He is on the editorial board of the International Journal of Computer Vision, and the Journal of Computer Vision and Graphics. He was selected as a Distinguished Lecturer of IEEE SP Society for the period of 2000-2001. He has received several awards, including the David Marr Prize in computational vision, and IEEE R\&A K-S Fu Memorial Best Transaction Paper Award. In addition, in 1992, his paper, "Numerical Shape from Shading and Occluding Boundaries," was selected as one of the most influential papers to have appeared in the Artificial Intelligence Journal within the past 10 years. He is a fellow of the IEEE. 